\title{
Hepatoid adenocarcinoma of the lung without production of $\alpha$-fetoprotein: A case report and review of the literature
}

\author{
JI NING SUN ${ }^{1}$, BAO LONG ZHANG ${ }^{2}$, LIN KUN LI $^{1}$, HAI YAN YU $^{1}$ and BIN WANG ${ }^{3}$ \\ ${ }^{1}$ Department of Radiology, Weifang People's Hospital, Weifang, Shandong 261041; \\ ${ }^{2}$ Department of Radiology, Weifang Maternity and Child Care Hospital, Weifang, Shandong 261011; \\ ${ }^{3}$ Medical Imaging Research Institute, Binzhou Medical University, Yantai, Shandong 264003, P.R. China
}

Received February 11, 2015; Accepted March 22, 2016

DOI: $10.3892 / \mathrm{ol} .2016 .4559$

\begin{abstract}
Hepatoid adenocarcinoma of the lung (HAL) is a rare type of extrahepatic hepatoid adenocarcinoma. The majority of such cases that have been reported in the English literature have demonstrated elevated serum $\alpha$-fetoprotein (AFP) and/or positive AFP expression on histopathological analysis, and have had poor prognoses. The current study reports an extremely rare case of HAL in the right upper lung without AFP production in a 59-year-old male patient. The patient underwent radical right upper lobectomy via video assisted thoracoscopic surgery. No signs of local recurrence or distant metastasis have been observed for 23 months after the surgery. The serum AFP level following surgery remained within the normal range. Although the diagnosis of HAL predominantly depends on pathological examination, a review of the literature demonstrates that HAL has certain characteristic features with regard to its clinical and computed tomography findings and prognoses, and clinicians should be aware that clinicians should be aware that monitoring serum AFP levels, as well as CT findings, remains important.
\end{abstract}

\section{Introduction}

Hepatoid adenocarcinoma is a relatively rare extrahepatic tumor with morphological features similar to hepatocellular carcinoma (1). The most common site of origin for hepatoid adenocarcinoma is the stomach $(83.9 \%)(2-4)$. Hepatoid carcinoma of the lung (HAL) is rare, and its incidence accounts for $2.3 \%$ of hepatoid adenocarcinoma cases (1). The biological significance of HAL remains to be elucidated (4), and its clinical symptoms are generally atypical. Although

Correspondence to: Professor Bin Wang, Medical Imaging Research Institute, Binzhou Medical University, 346 Guanhai Road, Laishan, Yantai, Shandong 264003, P.R. China

E-mail: binwang001@aliyun.com

Key words: hepatoid adenocarcinoma, lung, $\alpha$-fetoprotein, computed tomography computed tomography (CT) images may provide some indications for a diagnosis of HAL, confirmation of this diagnosis requires morphological and immunohistochemical confirmation. Previous studies have suggested that HAL has a poorer prognosis compared with more common types of lung tumor (5-7). In general, the prognosis of HAL appears poor and its invasiveness may explain its high mortality rate $(1,7)$. The overwhelming majority of cases reported in the English literature have presented with elevated serum $\alpha$-fetoprotein (AFP) levels and/or positive AFP expression on histopathological analysis, and were commonly associated with poor prognosis (8). Furthermore, based on the present literature review, it appears that pathological state is the most significant prognostic factor. The present study reports a case of HAL without AFP production in which the patient underwent radical right upper lobectomy via single-port video assisted thoracoscopic surgery (VATS). A description of the clinical and pathological findings, chest CT images, the outcome following surgery, and a review of the relevant literature are presented.

\section{Case report}

In April 2014, a 59-year-old man with a history of mild alcohol consumption, tobacco smoking (ceased 9 years previously) and diabetes for 8 years sought medical consultation at Weifang People's Hospital (Weifeng, China) for a productive cough without chest pain or dyspnea. The patient did not have alcoholic hepatitis or any remarkable relevant family medical history.

Non-contrast CT images (SOMATOM Definition AS; Siemens Healthcare, Erlangen, Germany) revealed a well-defined mass, of which the longest cross-sectional dimension was $4.5 \times 3.8 \times 3.5 \mathrm{~cm}$, in the right upper lobe of the lung (Fig. 1A and B). The mass extended to the pleura, causing thickening. Following intravenous injection of contrast agents, the tumor appeared inhomogeneous and the majority of the tumor exhibited mild enhancement, and moderate to significant peripheral and patchy enhancement were found after a delay (Fig. 1C-F). No enlarged lymph nodes were detected in the hila or mediastinum. The patient underwent percutaneous lung biopsy under CT guidance and routine paraffin sectioning and hematoxylin and eosin 
A

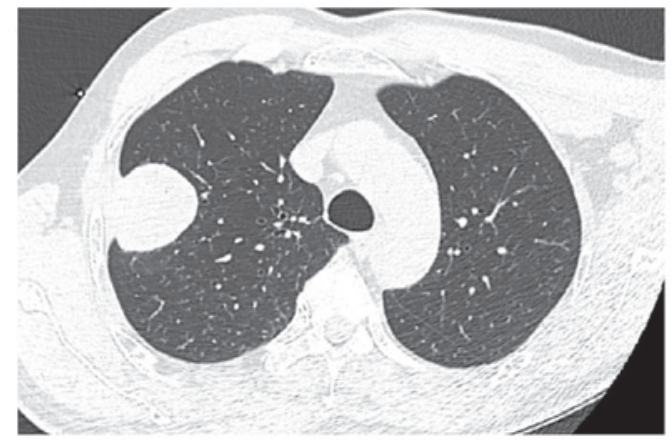

C

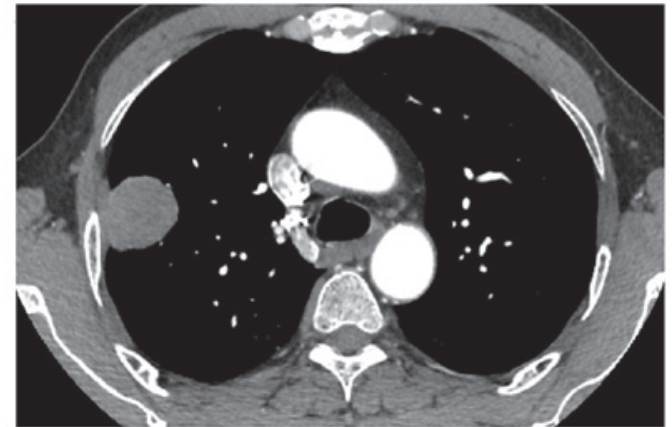

$\mathbf{E}$

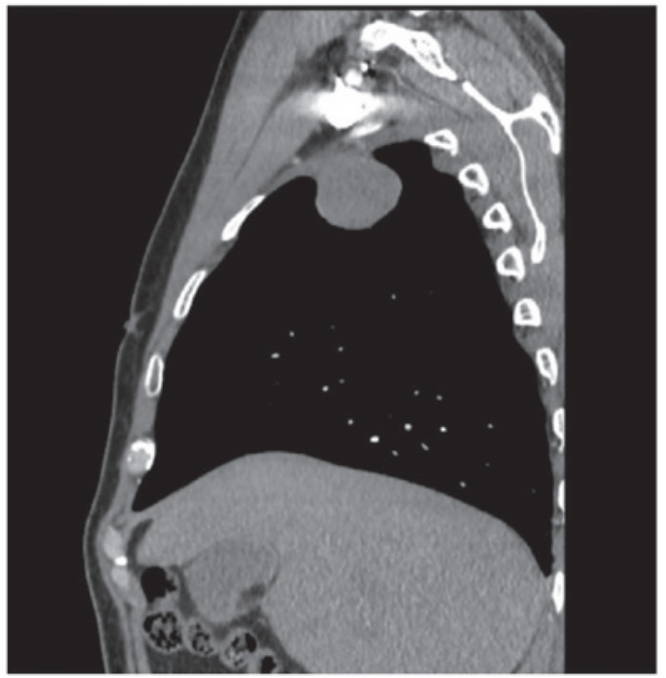

B

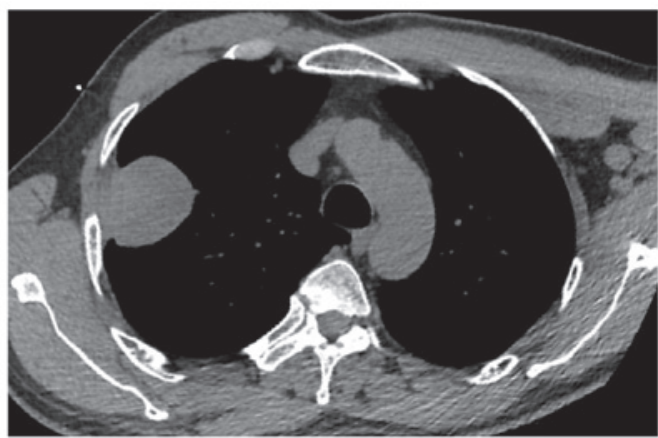

$\mathbf{D}$

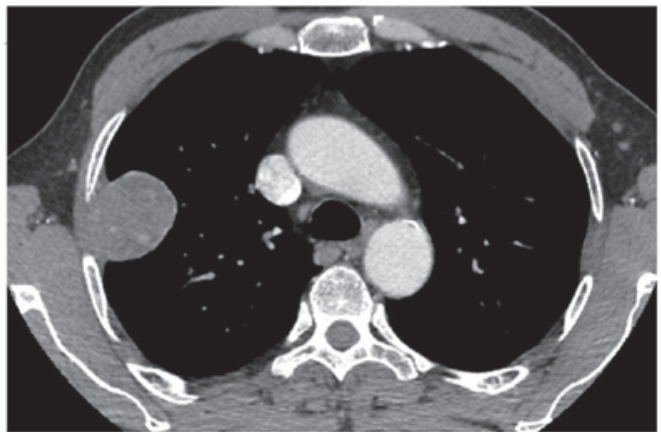

$\mathbf{F}$

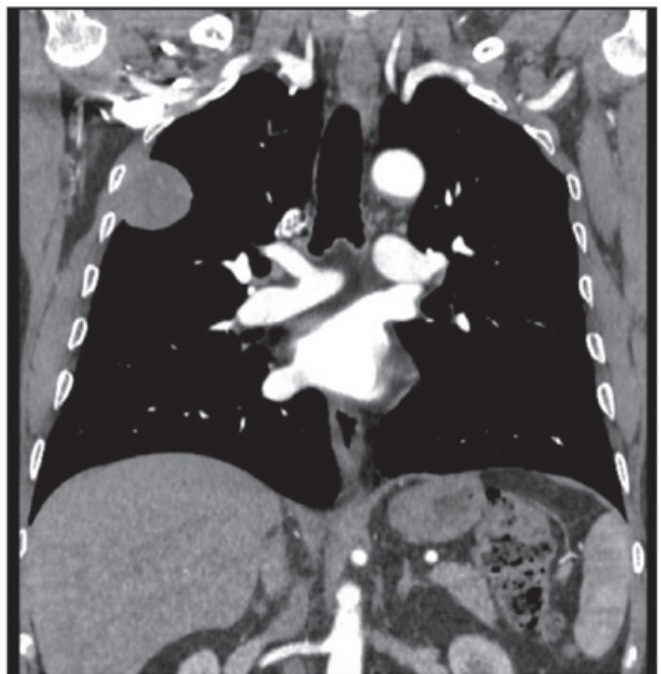

Figure 1. Chest CT images from a 59-year-old male patient. (A) CT image of the lung window revealed a well-defined mass in the right upper lung. (B) Non-contrast axial CT image of the mediastinal window revealed a well-defined mass invading the costal pleura. (C) Contrast-enhanced CT image in the arterial phase revealed inhomogenous mild enhancement in the majority of the tumor. (D) Contrast-enhanced CT image in the delay phase revealed moderate to significant peripheral and patchy enhancement. (E and F) Contrast-enhanced sagittal and coronal plane CT images tumor. CT, computed tomography.

A

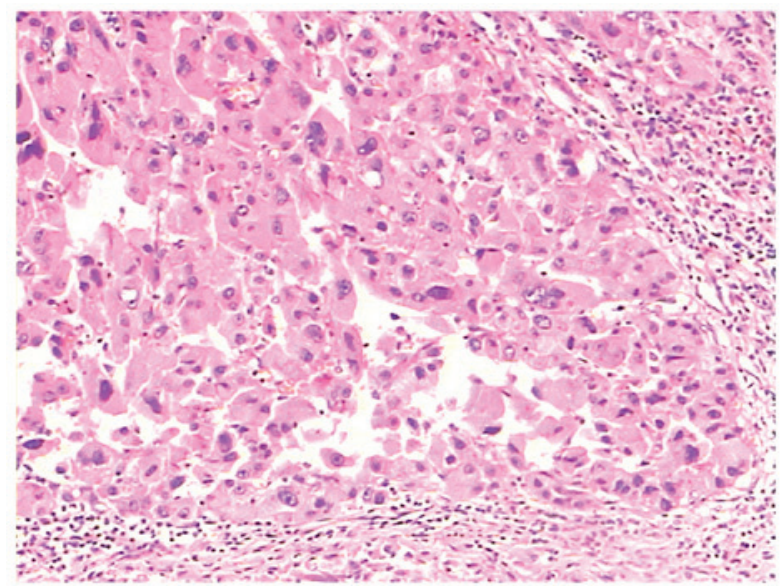

B

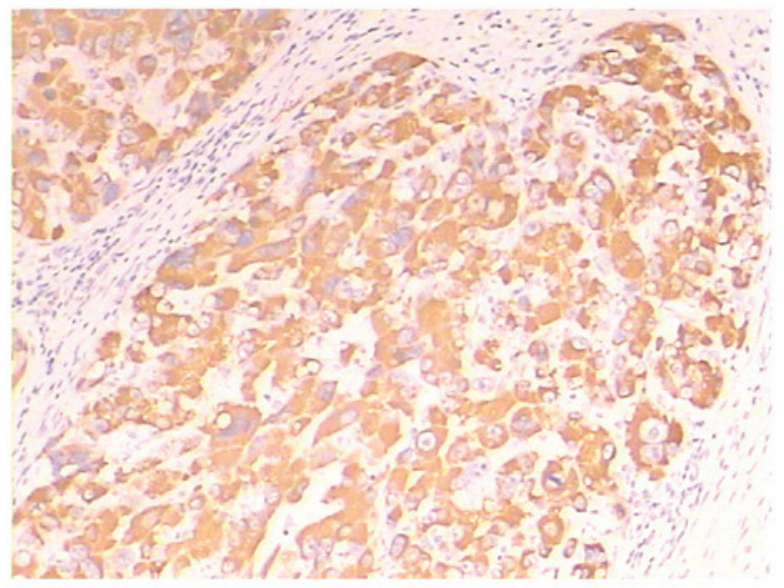

Figure 2. Histological findings. (A) The cells were arranged in trabecular and solid mass-like growth patterns (hematoxylin and eosin staining; magnification, x10). (B) Immunohistochemical staining revealing positivity for hepatocyte paraffin 1. 


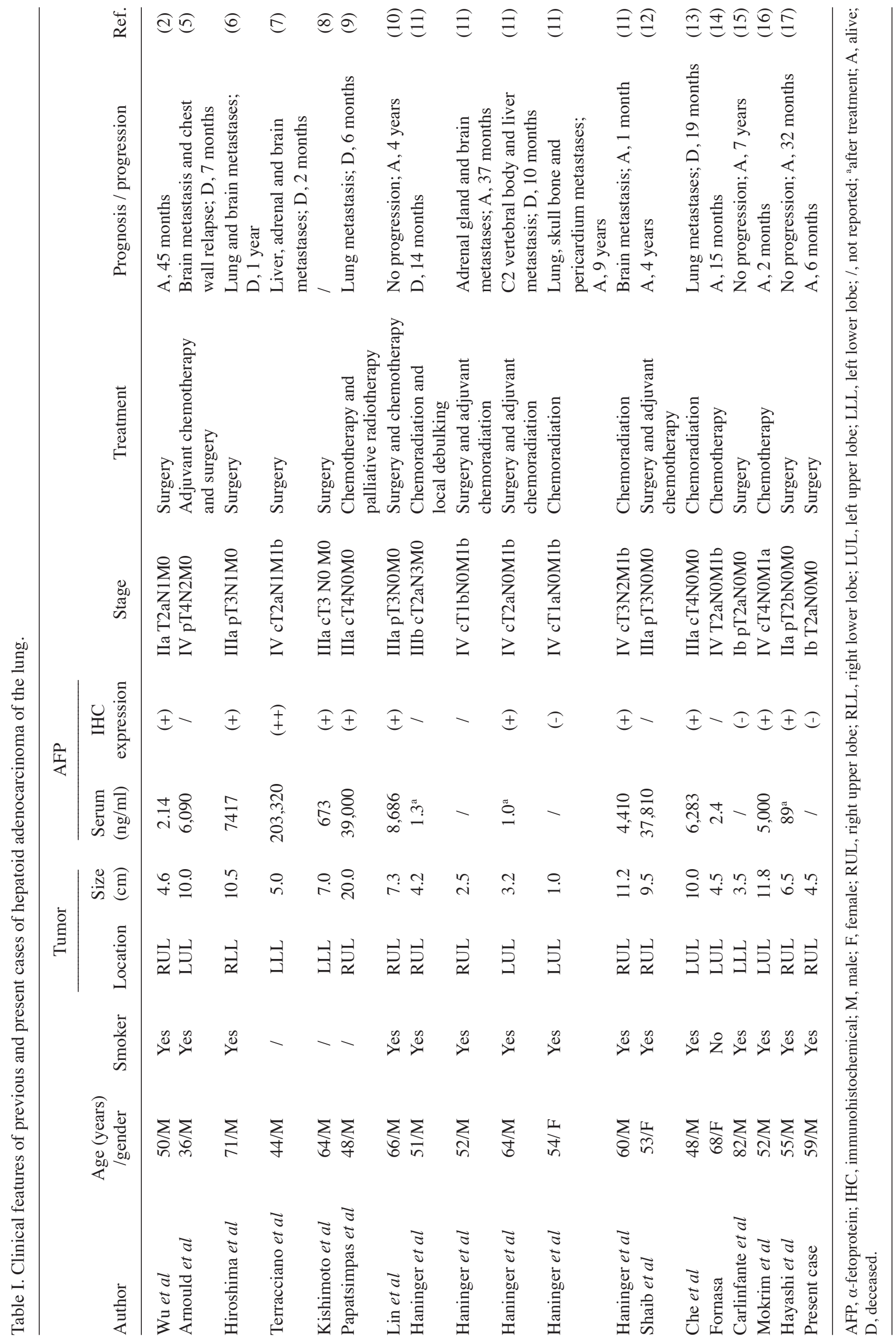


staining were used to detect the pathomorphological changes, which revealed adenocarcinoma. According to the findings above, the clinical stage of the patient was diagnosed as $\mathrm{Ib}$ (T2aN0M0). No abnormality was found in the blood cell count or biochemistry. With regard to tumor markers, carcinoembryonic antigen, CY211 and neuron-specific enolase levels were observed to be normal. Serum AFP level was not examined.

The patient was admitted for a pneumectomy of the right upper lobe via single-port VATS. During the surgery, a $4 \times 3 \times 3 \mathrm{~cm}$ mass with adhesion to the chest wall in the apical segment of right upper lobe was detected, along with enlarged subcarinal and hilar lymph nodes. A right upper lobectomy with dissection of multiple lymph nodes was successfully performed.

Pathological examination of the specimen revealed that the tumor measured $4.5 \times 3.5 \times 3.5 \mathrm{~cm}$ on its largest cross-section, and additionally exhibited extensive necrosis. Histologically, staining with hematoxylin and eosin (Fig. 2A) of formalin-fixed paraffin-embedded 4- $\mu \mathrm{m}$ thick tumor tissues under a microscope (BX43; Olympus Corporation, Tokyo, Japan) using a DAB Peroxidase Substrate kit (Vector Laboratories, Inc., Burlingame, CA, USA) revealed that the tumor was composed of poorly differentiated cancer cells with abundant eosinophilic cytoplasm and round nuclei, resembling hepatocellular carcinoma. The cells were arranged in trabecular and solid mass-like growth patterns. The tissue sections were immunostained with the following primary antibodies: Monoclonal mouse anti-hepatocyte (clone OCH1E5; dilution, 1:200; catalog. no., IS62430; Dako, Glostrup, Denmark); monoclonal mouse anti-cluster of differentiation (CD)34 (dilution, 1:200; catalog no., ab30375; Abcam, Cambridge, MA, USA); and monoclonal rabbit anti-CD10 (dilution, 1:200; catalog no., 790-4506; Roche Diagnostics, Indianapolis, IN, USA), overnight at $4^{\circ} \mathrm{C}$. The samples were subsequently incubated with horseradish peroxidase-conjugated secondary antibody (goat anti-mouse; catalog no., A0216; goat anti-rabbit; catalog no., A0208; both dilution, 1:500; both from Beyotime Institute of Biotechnology, Haimen, China) for $30 \mathrm{~min}$ at $37^{\circ} \mathrm{C}$. Immunohistochemical staining was positive for hepatocyte paraffin 1 (Fig. 2B), CD10 and CD34 in the vessels; AFP, thyroid transcription factor-1, cytokeratin 8/18, CD56, chromogranin A, synaptophysin and napsin A were negative. The Ki-67 score was observed to be $20 \%$. The tumor had invaded the lung capsule; however, all sampled lymph nodes were observed to be free of tumor involvement. Consequently, the histological type was determined to be hepatoid adenocarcinoma, and the pathological stage was determined to be Ib (pT2aN0M0).

The patient received regular outpatient follow-up and no local recurrence or distant metastasis was found after 23 months. The serum AFP level remained within the normal range. It is expected that long-term survival can be expected in the present case, although careful follow-up observations are required.

Written informed consent was obtained from the patient for publication of the present case report and any accompanying images. The present study was approved by the Medical Ethics Committee of Weifang People's Hospital (Weifang, China).

\section{Discussion}

HAL is an extremely rare tumor that was first described by Ishikura et al (3). Subsequent reports in case series or single case reports have described the clinical manifestations and pathological features of HAL; however, to the best of our knowledge, little has been discussed regarding its CT findings, with the exception of one paper (2). Thus, the present study aimed to enrich the available information regarding clinical findings, imaging characteristics and treatment protocols of HAL and to ascertain their associations with prognosis. A total of 18 cases were identified from 14 reports in the English literature with available full texts on HAL confirmed by histopathology. These cases, in addition to the present case, were reviewed, and the clinical findings, imaging features and prognosis were extracted for analysis (Table I).

Clinically, patients with HAL typically presented with nonspecific symptoms, including chest pain, back pain, shooting pain in the shoulder, cough and dyspnea, asthenia, anorexia and weight loss. The ages at diagnosis ranged from 36 to 82 years. Smoking history was available in 16 cases, of which 15 had a history of tobacco smoking. In the reviewed cases, the incidence of HAL was markedly higher in men than in women (16 vs. 3 cases; male to female ratio, 5.3:1); notably, no significant gender predominance has been identified in reports on hepatoid adenocarcinoma of the stomach (4). The size of the HAL masses reviewed herein was variable, ranging from 1.0 to $20.0 \mathrm{~cm}$.

In the majority of cases of HAL described in the literature (5-13), HAL was considered to be associated with positive AFP expression on histopathology and/or elevated serum AFP. However, not all HALs produced AFP: The serum AFP levels of 2 cases $(2,14)$ were within the normal ranges prior to surgery, and immunohistochemical AFP expression was negative in 3 cases, including the present case $(11,15)$. These findings are consistent with the opinion of Haninger et al (11) that AFP expression is not requisite for diagnosis of HAL, and that the diagnosis depends on the recognition of characteristic histological features. Therefore, although no AFP expression was detected in present case, the diagnosis was still established based on morphology and immunophenotype.

In the reviewed literature, decreasing AFP level following surgical resection was observed in 7 cases $(5,6,9,12,13,16,17)$, and, in 3 cases $(5,9,13)$, the level was elevated markedly when the patient relapsed. Thus, the serum AFP level appears to be a reliable indicator to monitor disease response to therapy, relapse and aggravation, and this may be identified much earlier than any indication from imaging findings (18).

Although CT images were only provided in 7 cases in the literature review $(2,9,11,16)$, in addition to the present case, certain information was also obtained indirectly from a description of CT features and/or pathological specimens (11 cases). These data indicated the following findings. Firstly, the location of the tumor was characteristic, with the majority (15/18 cases) located in the upper lung fields, and a tendency to be adjacent to the costal pleura (5 cases) or mediastinal pleura ( 6 cases); the former often adhered to the chest wall, while paramediastinal HALs were adjacent to blood vessels, meaning that it was easy 
for them to invade the structures of the homolateral mediastinum (including the trachea and esophagus) invasion. Secondly, whilst none of the imaging features is considered typical enough to diagnose HAL directly, certain findings should be regarded as concerning. HAL tumors usually appeared inhomogeneous, and large necrotic areas may be detected in the tumor and also observed by pathological examination $(2,5,6,9-11,15,17)$; this is consistent with the report by $\mathrm{Wu}$ et al (2). Following contrast enhancement, the tumors exhibited a mild to moderate inhomogeneous enhancement and the necrotic areas appeared more clearly. In summary, imaging evaluation serves a limited but useful role in the selection of a therapeutic schedule for HAL, as it is able to detect lymph node or distant metastases and help to assess the clinical stage. Knowledge of HAL imaging characteristics is evolving and may in future indicate associations with prognosis.

The limitations of the published literature for establishing prognostic indicators included the limited number of patients, short follow-up time and variable disease stages at diagnosis. Generally, HAL is considered to be associated with a poor prognosis $(5,7,9)$. However, prognosis is likely to be associated with clinical stage; as reported by Carlinfante et al (15), one patient had a good prognosis (7 years survival) after having a tumor with small dimensions and without extension outside of the lung (stage T2aN0M0). The significance of staging is also indicated by the cases of stage IIa (pT2bN0M0) and stage IIa (T2aN1M0) disease, as reported by Hayashi et al (17) and Wu et al (2), in which the tumors were successfully cured by local excision and no signs of recurrence and metastasis were observed after 32 and 45 months post surgery, respectively. In the reviewed literature, the cases receiving surgery and/or chemoradiation, even at an intermediate to advanced stage, also showed favorable prognoses (10-12). These findings suggest that clinical stage represents a significant prognostic factor in HAL, but that curative resection and reasonable adjuvant chemoradiation also have a substantial influence on the prognosis of HAL at these stages (9).

It was noteworthy that a female patient with stage IV disease (cT1aN0M1b) with negative AFP expression had the longest survival time of 9 years out of all cases followed up, and the metastatic lesion in this patient was controlled completely by chemoradiation. AFP expression in the case reported by Carlinfante et al (15) was also negative. These two cases support the opinion of Papatsimpas et al (9) that the patients with normal AFP levels at presentation tended to have a better overall survival time, even after relapse; this is likely to represent a different subset of HAL with better biological behavior. Based on the above analysis, we considered that long-term survival could be expected in our case, although close follow-up was undertaken.

HAL is a rare tumor that is difficult or impossible to distinguish from other malignant entities in the lung by imaging alone $(2,5,15,17)$. The number of recorded cases in the English literature, including the present case, is small, and any firm conclusions cannot be drawn; however, a mass in the lung located adjacent to the costal or mediastinal pleura, with a large necrotic region and mild to moderate inhomogeneous enhancement, together with elevated serum AFP levels, should indicate the possibility of HAL if there is no lesion present in the liver $(2,17)$.
In conclusion, the present study reported a case of HAL without AFP production in which the patient underwent radical right upper lobectomy via single-port VATS. The patient received regular outpatient follow-up and no local recurrence or distant metastasis was observed after 23 months. The serum AFP level remained within the normal range. In general, although HAL is usually associated with poor prognosis, for early stage HAL patents, radical surgical resection may markedly increase the cure rate $(3,15,17,19)$. Even for patients in whom lymph node or distant metastases are detected, palliative lobectomy and adjuvant chemoradiation are considered appropriate treatments to improve the survival time $(10-12,20)$. However, the optimal management of HAL is still not well defined and further investigations are required $(10-12,20)$. In addition, once the diagnosis of HAL is confirmed by immunohistochemistry, clinicians should be aware that monitoring serum AFP levels, as well as CT findings, remains important.

\section{References}

1. Su JS, Chen YT, Wang RC, Wu CY, Lee SW and Lee TY: Clinicopathological characteristics in the differential diagnosis of hepatoid adenocarcinoma: A literature review. World J Gastroenterol 19: 321-327, 2013.

2. Wu Z, Upadhyaya M, Zhu H, Qiao Z, Chen K and Miao F: Hepatoid adenocarcinoma: Computed tomographic imaging findings with histopathologic correlation in 6 cases. J Comput Assist Tomogr 31: 846-852, 2007.

3. Ishikura $\mathrm{H}$, Kanda $\mathrm{M}$, Ito $\mathrm{M}$, Nosaka $\mathrm{K}$ and Mizuno $\mathrm{K}$ : Hepatoid adenocarcinoma: A distinctive histological subtype of alpha-fetoprotein producing lung carcinoma. Virchows Arch A Pathol Anat Histopathol 417: 73-80, 1990.

4. Nagai E, Ueyama T, Yao T and Tsuneyoshi M: Hepatoid adenocarcinoma of the stomach. A clinicopathologic and immunohistochemical analysis. Cancer 72: 1827-1835, 1993.

5. Arnould L, Drouot F, Fargeot P, Bernard A, Foucher P, Collin F and Petrella T: Hepatoid adenocarcinoma of the lung: Report of a case of an unusual alpha-fetoprotein-producing lung tumor. Am J Surg Pathol 21: 1113-1118, 1997.

6. Hiroshima K, Iyoda A, Toyozaki T, Haga Y, Baba M, Fujisawa T, Ishikura $\mathrm{H}$ and Ohwada $\mathrm{H}$ : Alpha-fetoprotein-producing lung carcinoma: Report of three cases. Pathol Int 52: 46-53, 2002.

7. Terracciano LM, Glatz K, Mhawech P, Vasei M, Lehmann FS, Vecchione R and Tornillo L: Hepatoid adenocarcinoma with liver metastasis mimicking hepatocellular carcinoma: An immunohistochemical and molecular study of eight cases. Am J Surg Pathol 27: 1302-1312, 2003.

8. Kishimoto T, Yano T, Hiroshima K, Inayama Y, Kawachi K and Nakatani Y: A case of *-fetoprotein-producing pulmonary carcinoma with restricted expression of hepatocyte nuclear factor- $4^{*}$ in hepatoid foci: A case report with studies of previous cases. Hum Pathol 39: 1115-1120, 2008.

9. Papatsimpas G, Kamposiorasa K, Goulab K, Papaparaskeva K, Loukides S, Kotoulas C, Kelekis N, Xiros N, Pectasides D and Koumarianou A: Hepatoid pancoast tumor. A case report and review of the literature. Lung Cancer 77: 239-245, 2012.

10. Lin SF, Hsu WH and Chou TY: Primary pulmonary hepatoid carcinoma: Report of a case and review of the literature. Kaohsiung J Med Sci 29: 512-516, 2013.

11. Haninger DM, Kloecker GH, Bousamra Ii M, Nowacki MR and Slone SP: Hepatoid adenocarcinoma of the lung: Report of five cases and review of the literature 27: 535-542, 2014.

12. Shaib W, Sharma R, Mosunjac M, Farris AB III and El Rayes B: Hepatoid adenocarcinoma of the lung: A case report and review of the literature. J Gastrointest Cancer 45 (Suppl 1): 99-102, 2014.

13. Che YQ, Wang S, Luo Y, Wang JB and Wang LH: Hepatoid adenocarcinoma of the lung: Presenting mediastinal metastasis without transfer to the liver. Oncol Lett 8: 105-110, 2014.

14. Fornasa F: Soft-tissue localization of hepatoid adenocarcinoma: First case report. Case Rep Oncol 3: 212-217, 2010. 
15. Carlinfante G, Foschini MP, Pasquinelli G, Scotti R and Cavazza A: Hepatoid carcinoma of the lung: A case report with immunohistochemical, ultrastructural and in-situ hybridization findings. Histopathology 37: 88-89, 2000.

16. Mokrim M, Belbaraka R, Allaoui M, Kairaouani M, Mahassini N, Tahri A and Errihani H: Hepatoid adenocarcinoma of the lung: A case report and literature review. J Gastrointest Cancer 43 (Suppl 1): 125-127, 2012.

17. Hayashi Y, Takanashi Y, Ohsawa H, Ishii H and Nakatani Y: Hepatoid adenocarcinoma in the lung. Lung Cancer 38 211-214, 2002.
18. Furukawa H, Oda K, Nishie K, Miyake K, Ota K, Nishida K, Munakata T, Ikeda H, Onaru K and Goma I: A case of alpha-fetoprotein-producing recurrent lung adenocarcinoma successfully treated with radiation therapy (high-grade adenocarcinoma of fetal lung type). Gan To Kagaku Ryoho 39: 2393-2395, 2012 (In Japanese).

19. Slotta JE, Jüngling B, Kim YJ, Wagner M, Igna D and Schilling MK: Hepatoid adenocarcinoma of the transverse colon. Int J Colorectal Dis 27: 989-991, 2012.

20. Kitada M, Ozawa K, Sato K, Matsuda Y, Hayashi S, Tokusashi Y, Miyokawa N and Sasajima T: Alpha-fetoprotein-producing primary lung carcinoma: A case report. World J Surg Oncol 9: 47, 2011. 